\title{
COMMENT
}

\section{Erythropoietin and neonatal treatment: still more questions than answers}

\author{
Gunnar Naulaers ${ }^{1,2}$ \\ Pediatric Research (2018) 84:793-794; https://doi.org/10.1038/s41390-018-0213-7
}

In this issue Teramo and Widness ${ }^{1}$ describe the different pathways of production of erythropoietin (EPO) in the fetus and placenta during pregnancy and how EPO works as a protective agent for the foetal and neonatal brain and gut. A good overview is given about what we know, and certainly also what we do not know about EPO and its importance in foetal and neonatal hypoxia. This review suggests further research in different domains of foetal and neonatal medicine.

The authors describe the studies showing that EPO is a biomarker of both hypoxic and anaemic hypoxia in different pathological situations in pregnancy. Studies in sheep showed that during the foetal period an increase in EPO is caused by placental production, which is much higher than the renal production during normoxia and only starts when the foetus becomes hypoxic. Although several studies have shown an association between high levels of EPO in amniotic fluid and cord blood and foetal acidosis, as well as poor neonatal outcomes, ${ }^{1}$ no studies have been performed regarding the EPO levels in umbilical blood and late (neurodevelopmental outcome at 2 years) outcome variables. More research is needed to clarify the clinical role of foetal EPO as an intrauterine hypoxia marker.

The neuroprotective effect of EPO as an anti-apoptotic and antiinflammatory agent has been described through different mechanisms. Furthermore, EPO enhances angiogenesis and neurogenesis in the brain. ${ }^{2}$ The neuroprotective effects of EPO in both preterm infants and term infants with hypoxic ischaemic encephalopathy (HIE) have been studied; however, there is still a lot of debate regarding the right dose and timing.

An overview of different pharmacokinetic studies in HIE, also during cooling, is given, which shows that doses of $1000 \mathrm{U} / \mathrm{kg}$ result in adequate blood levels of EPO in the blood. Although neuroprotective studies are well described, I agree that it remains an important question when EPO needs to be started to have an additional neuroprotective effect and whether umbilical or amniotic fluid levels at birth might help in adjusting the postnatal EPO dose. Individual treatment according to these levels might be a possible future strategy. If we look at the published studies, there is still no consensus on how long EPO needs to be given to have long-lasting neuroprotective effects. In preterm infants, it is interesting that the study of Song et al., where EPO was given for 2 weeks, $^{3}$ yielded more effect on the outcome than the study by Natalucci et al., where three doses of EPO were given in the first $42 \mathrm{~h}^{4}$ This suggests that EPO needs to be given long enough to have a long-lasting effect. A study in rodents showed a neuroprotective effect after transient neonatal stroke when the first dose was given 7 days later. ${ }^{5}$ This leads to opportunities for treatment of cerebral lesions that are diagnosed in the first postnatal days like intraventricular bleedings in preterm infants and neonatal stroke in term infants. The EpoRepair study (NCT02076373) is currently looking at the effect of EPO at a dose of $2000 \mathrm{U} / \mathrm{kg}$ starting at day 5 in preterm infants with an intraventricular bleeding grade of 2 or more. Other, different studies are ongoing in preterm infants with white matter injury and term infants with hypoxic encephalopathy and neonatal stroke. ${ }^{2,6}$ These studies will further define the right indications, dose and timing for using EPO as a neuroprotective agent.

Another important question raised in this review is whether EPO can be given orally. Although the different studies regarding the use of oral EPO as treatment for anaemia are not conclusive, erythropoietin protects epithelial cells from excessive autophagy and apoptosis. The authors describe the protective effect of high levels of EPO in the amniotic fluid on necrotizing enterocolitis (NEC) and suggest that the high levels of EPO in breast milk might also explain partly its protective effect for NEC. Whether adding EPO orally in babies who receive artificial milk acts as a prevention for NEC has not been studied. Interestingly, no information about the use of breastmilk is given in the different clinical studies for neuroprotection, which again suggests that it would be good to monitor EPO levels in the blood during these studies.

Although the different theories, based on the current research, remain hypothetical, this review certainly must stimulate to develop further research on the production and treatment options of EPO as a protective agent in hypoxic conditions for both the foetus and the neonate. The differences in dose, timing and length of the therapy in the different studies will make it difficult to develop a good treatment plan for the different indications. The ongoing studies will help us to get a better view on these questions and hopefully lead to effective use of EPO in neonates.

\section{ADDITIONAL INFORMATION}

Competing interests: The authors declare no competing interests.

Publisher's note: Springer Nature remains neutral with regard to jurisdictional claims in published maps and institutional affiliations.

\section{REFERENCES}

1. Teramo, K. A. \& Widness, J. A. Increased fetal plasma and amniotic fluid erythropoietin concentrations: markers of intrauterine hypoxia. Neonatology 95, 105-116 (2009).

2. Juul, S. E. \& Pet, G. C. Erythropoietin and neonatal neuroprotection. Clin. Perinatol. 42, 469-481 (2015).

\footnotetext{
${ }^{1}$ Department of Development and Regeneration, KU Leuven, Leuven, Belgium and ${ }^{2}$ Neonatal Intensive Care Unit, University Hospitals Leuven, Leuven, Belgium
} Correspondence: Gunnar Naulaers (gunnar.naulaers@uzleuven.be)

Received: 24 April 2018 Revised: 4 October 2018 Accepted: 9 October 2018

Published online: 23 October 2018 
Erythropoietin and neonatal treatment: still more questions than answers

G Naulaers

794

3. Song, J. et al. Recombinant human erythropoietin improves neurological outcomes in very preterm infants. Ann. Neurol. 80, 24-34 (2016).

4. Natalucci, G. et al. Effect of early prophylactic high-dose recombinant human erythropoietin in very preterm infants on neurodevelopmental outcome at 2 years: a randomized clinical trial. JAMA 315, 2079-2085 (2016).
5. Larpthaveesarp, A., Georgevits, M., Ferriero, D. M. \& Gonzalez, F. F. Delayed erythropoietin therapy improves histological and behavioral outcomes after transient neonatal stroke. Neurobiol. Dis. 93, 57-63 (2016).

6. Benders, M. J. et al. Feasibility and safety of erythropoietin for neuroprotection after perinatal arterial ischemic stroke. J. Pediatr. 164, 481-486 (2014). 